\title{
A switching synchronization scheme for a class of chaotic systems
}

\author{
Ömer Morgül, Murat Akgül \\ Bilkent University, Department of Electrical and Electronics Engineering, 06533, Bilkent, Ankara, Turkey \\ Received 23 October 2001; accepted 10 July 2002 \\ Communicated by A.P. Fordy
}

\begin{abstract}
In this Letter, we propose an observer-based synchronization scheme for a class of chaotic systems. This class of systems are given by piecewise-linear dynamics. By using some properties of such systems, we give a procedure to construct the gain of the observer. We prove various stability results and comment on the robustness of the proposed scheme. We also present some simulation results.
\end{abstract}

(C) 2002 Elsevier Science B.V. All rights reserved.

PACS: $05.45 . \mathrm{G}$

Keywords: Chaos; Synchronization; State observers; Stabilization; Robustness

\section{Introduction}

Although the concept of synchronization of chaotic systems may seem somewhat paradoxical, it has been known since the seminal work [1] that it is possible, and even more surprisingly this property is robust in certain cases, see, e.g., [2]. In recent years, many aspects of chaotic dynamics including synchronization and control of chaotic systems have received considerable attention among scientists in many different fields. The literature is quite rich on this subject, and interested reader may consult to, e.g., $[3,4]$.

Most of the synchronized chaotic systems consist of two parts: a generator of chaotic signals (drive system), and a receiver (response system). The response system is usually a duplicate of a part (or the whole) of the drive system. A chaotic signal generated by the

E-mail address: morgul@ee.bilkent.edu.tr (Ö. Morgül). drive system, which is called the synchronization signal, is usually transmitted to the response system to achieve the synchronization. One of the motivations for the synchronization is the possibility of sending messages through chaotic systems for secure communication, see [5].

Various synchronization schemes are proposed in the literature, see, e.g., [4], and in most of these works a systematic procedure to determine the response system and the synchronization signal is not given. A particular synchronization scheme which utilizes such a systematic procedure is the observer-based synchronization scheme, see, e.g., [2,6,7]. In this approach, typically the response system is a duplicate of the drive system, and a synchronization error term, which is the difference between the synchronization signal and a similar signal generated in the response system, is injected into the response system through a gain vector, which is called the observer gain. General procedures 
and conditions to determine this gain vector to guarantee synchronization for a given arbitrary drive system can be obtained, see, e.g., [2]. However, if the properties of a given particular drive system are not taken into consideration, the required gain may be quite high, see, e.g., [8]. High gain is not desired especially when the synchronization signal is corrupted with noise, as the noise will also be amplified by the gain. Also high gain values may cause large transients, and this might cause saturation in certain cases. One way to eliminate the high gain values is to incorporate the system properties into the observer-based design. In this approach, a particular class of systems may be considered and the observer-based approach could be modified for this particular class of systems.

In this Letter we will consider a special class of chaotic systems, which are characterized by piecewiselinear dynamics. Although the resulting dynamics seems to be rather restricted, nevertheless this class of systems contains many chaotic systems, including most of the chaotic electronic oscillators, already proposed in the literature. For this class of systems, we propose two observer-based synchronization schemes. We prove various synchronization results and comment on the robustness of the proposed schemes.

This Letter is organized as follows. In Section 2, we introduce the class of systems under consideration. In Section 3, we propose our synchronization schemes. We also prove various synchronization and stability properties, and comment on the robustness of the proposed schemes. In Section 4, we give various simulation results which indicate the effectiveness of the proposed schemes. Finally we give some concluding remarks.

\section{Problem statement}

In this Letter we will consider a special class of chaotic systems given by piecewise-linear dynamics. To be specific, let $-\infty<k_{1}<k_{2}<\cdots<k_{m-1}<\infty$ be given constants, and set $k_{0}=-\infty, k_{m}=\infty$, where $m \geqslant 2$. We define the regions $\mathcal{R}_{i} \in \mathbf{R}$ as

$\mathcal{R}_{i}=\left\{x \in R \mid k_{i-1} \leqslant x \leqslant k_{i}\right\}, \quad i=1,2, \ldots, m$.

We consider the systems given as:

$\dot{z}=f(z)$, where $z=\left(z_{1} \ldots z_{n}\right)^{\mathrm{T}} \in \mathbf{R}^{n}$, here the superscript $\mathrm{T}$ denotes the transpose, and $f: \mathbf{R}^{n} \rightarrow \mathbf{R}^{n}$ is a piecewise-linear map defined as:

$f(z)=A_{i} z+b_{i}, \quad z_{1} \in \mathcal{R}_{i}, \quad i=1, \ldots, m$.

Here, for $i=1, \ldots, m, A_{i} \in \mathbf{R}^{n \times n}$ are constant matrices and $b_{i} \in \mathbf{R}^{n}$ are constant vectors. To ensure the existence and continuity of the solutions of (2), we will assume that $f$ is a continuous function. This requirement puts some conditions on $A_{i}$ and $b_{i}$, some of which will be exploited in designing the synchronization schemes. These continuity requirements can easily be obtained by using $A_{i} z+b_{i}=A_{i+1} z+b_{i+1}$ for $i=1, \ldots, m-1, z_{1}=k_{i}$, and $z \in \mathbf{R}^{n}$ is arbitrary otherwise. After some straightforward algebra, we see that the following holds

$A_{i+1}=A_{i}+\hat{h}_{i} e_{1}^{\mathrm{T}}, \quad i=1, \ldots, m-1$,

for some vectors $\hat{h}_{i} \in \mathbf{R}^{n}$, here $e_{1}$ is the first unit vector, i.e., $e_{1}=(10 \ldots 0)^{\mathrm{T}} \in \mathbf{R}^{n}$. In other words, $A_{i+1}$ and $A_{i}$ only differs in their first columns. Hence, there exist a constant matrix $A \in \mathbf{R}^{n \times n}$ and constant vectors $h_{i} \in \mathbf{R}^{n}$ such that the following holds:

$A_{i}=A+h_{i} e_{1}^{\mathrm{T}}, \quad i=1, \ldots, m$.

We note that this representation is not unique, since $h_{i} e_{1}^{\mathrm{T}}$ only affects the first column of $A$.

For synchronization, we need a synchronization signal to be sent to the receiver. A natural choice for the synchronization signal is $y=z_{1}$. Note that in the context of observer-based synchronization schemes, we have the following output function for the system (2)

$y=C z, \quad C=e_{1}^{\mathrm{T}}$.

As is customary in observer-based schemes, we assume that the pair $(C, A)$ is observable, i.e., the following matrix $Q$ is nonsingular:

$Q=\left(\begin{array}{c}C \\ C A \\ \vdots \\ C A^{n-1}\end{array}\right)$.

see, e.g., $[2,6,7]$.

The class of systems which could be described by the equations given above might be limited. However, there are meaningful classes of chaotic systems which 
could be represented in this framework. Such a class is the Lur'e type systems which are frequently encountered and investigated in the literature, see, e.g., $[2,8-$ 10], and the references therein. These systems can be represented as

$\dot{z}=A_{L} z-b_{L} f(y), \quad y=C_{L} z$,

where $A_{L} \in \mathbf{R}^{n \times n}, b_{L}, C_{L}^{\mathrm{T}} \in \mathbf{R}^{n}$ and $f: \mathbf{R} \rightarrow \mathbf{R}$ is an arbitrary function. Let us assume that $f(\cdot)$ is given by a piecewise-linear characteristics as

$f(y)=c_{i} y+d_{i}, \quad y \in \mathcal{R}_{i}, \quad i=1, \ldots, m$,

where $c_{i}, d_{i}$ are scalar constants. By using (9) in (8) we obtain the structure given by (2), (3) with

$A_{i}=A_{L}-c_{i} b_{L} C_{L}, \quad b_{i}=-d_{i} b_{L}$.

If $y=z_{1}$, i.e., $C_{L}=e_{1}^{\mathrm{T}}$, then (6) is also satisfied. If $y \neq z_{1}$, then by using the coordinate change $\tilde{z}=Q z$, where $Q$ is given by (7), with $C=C_{L}, A=A_{L}$, the system (8), (9) could be transformed into the form (2), (3) and (6).

Remark 1. If the nonlinearity $f(\cdot)$ is not piecewiselinear, then it may be approximated by a piecewiselinear one with any desired accuracy. If the original system exhibits a chaotic behaviour, and if the approximation error is sufficiently small, then it is reasonable to expect similar behaviour when the piecewiselinear approximation is used instead of the original $f(\cdot)$. However, the effect of this approximation error on the synchronization requires further investigation.

Remark 2. Most of the chaotic electronic oscillators proposed in the literature can be represented by the dynamics given above, see, e.g., [11,12] for more information on such oscillators. In particular, the wellknown Chua's chaotic oscillator, which is studied extensively in the literature, also belongs to such class of oscillators. This is particularly important, since it is known that Chua's oscillator is equivalent to a large class of chaotic systems already proposed in the literature, see, e.g., $[13,14]$.

\section{An observer-based synchronization scheme}

Consider the chaotic system given by (2), (3), (6). We assume that (5)-(7) also hold. For this system we first propose the following observer:

$\dot{\hat{z}}=A \hat{z}+h_{i} z_{1}+b_{i}+K\left(z_{1}-\hat{z}_{1}\right), \quad z_{1} \in \mathcal{R}_{i}$,

where $\hat{z} \in \mathbf{R}^{n}$ is the receiver state, and $K \in \mathbf{R}^{n}$ is a gain vector yet to be determined. Note that since $y=z_{1}$ is the synchronization signal, which is available, the observer structure given by (11) is realizable at the receiver.

Remark 3. To relate (11) with the observer-based synchronization scheme proposed in, e.g., [2], let us consider the following:

$\dot{\hat{z}}=A_{j} \hat{z}+b_{j}+u, \quad \hat{z}_{1} \in \mathcal{R}_{j}$,

where $j=1, \ldots, m$, and $u$ is an appropriate input to the observer. It is easy to show that if we choose the following $u$ for (12)

$u=h_{i} z_{1}+b_{i}-h_{j} \hat{z}_{1}-b_{j}+K\left(z_{1}-\hat{z}_{1}\right)$,

$z_{1} \in \mathcal{R}_{i}, \quad \hat{z}_{1} \in \mathcal{R}_{j}$,

then (12) reduces to (11). Hence, the synchronization scheme proposed in (11) is a special observer-based synchronization scheme. Note that in this formalism, the control action (13) can be interpreted as a set of "ifthen" rules given by: "if $z_{1} \in \mathcal{R}_{i}$ and $\hat{z}_{1} \in \mathcal{R}_{j}$, then $u$ is given by (13)". Hence, we have a set of rules, and the control action switches between them. This formalism could also be used in designing the so-called "fuzzy logic controllers" for such chaotic systems, see, e.g., [15]. Also note that there are different switching synchronization systems already proposed for some chaotic systems, see, e.g., [16].

To prove the synchronization property, let us define the synchronization error as $e=z-\hat{z}$. Upon differentiation and using (2), (11) we obtain:

$\dot{e}=(A-K C) e$.

Since the pair $(C, A)$ is assumed to be observable, the gain $K$ can be appropriately chosen so that $A-K C$ is stable (i.e., all eigenvalues are in the left half of the complex plane), hence the error $e$ decays exponentially to zero for any initial condition $e(0)=z(0)-\hat{z}(0)$. Moreover, the decay rate could be adjusted arbitrarily by proper choice of $K$, see $[2,6]$. 
Remark 4. Note that by using the techniques given in [2], one can also design other observer-based synchronization schemes for the systems given by (2). However, such schemes are for general nonlinear systems and particular structures of nonlinearities, e.g., the piecewise-linearity in present case, is not fully utilized. As a result, the required gain vectors in [2] usually depend on the Lipschitz constant $\gamma$ of the nonlinearity, and the largest gain would be proportional to $(n \gamma)^{n}$, see [17]. Usually $\gamma \gg 1$, and for large dimensions usually rather large gains are required to guarantee the synchronization. In the present case, however, the required gain $K$ is independent of any such Lipschitz constant, and the only requirement is the stability of $A-K C$. As a result, the synchronization is achieved with smaller gains. This is particularly important if the synchronization signal is corrupted by noise, since in this case the gain vector also multiplies the noise as well.

In (11), the synchronization signal enters into the observer dynamics through two ways. The first one is due to the switching rule $h_{i} z_{1}$, which may be considered as a nonlinear processing of $z_{1}$, and the second one is due to the linear injection term $K\left(z_{1}-\hat{z}_{1}\right)$. While the linear term is desirable and is present in many synchronization schemes, the nonlinear processing of synchronization signal is less desirable, especially when noise is present in the transmission of synchronization signal. One possible remedy is to use $\hat{z}_{1}$ in the nonlinear processing stated above. This observation leads to our second observer structure given as

$\dot{\hat{z}}=A_{i} \hat{z}+b_{i}+K\left(z_{1}-\hat{z}_{1}\right), \quad z_{1} \in \mathcal{R}_{i}$.

However, in this case the error dynamics will not be as simple as (14). In fact, by using (2), (3), (6) and (15) we obtain

$\dot{e}=\left(A_{i}-K C\right) e=F_{i} e, \quad z_{1} \in \mathcal{R}_{i}$,

where for simplicity we set $F_{i}=A_{i}-K C=A+$ $h_{i} C-K C$. To have a stable error, it is necessary that all $F_{i}$ be stable. Our next result states that this is possible by choosing $K$ appropriately.

Theorem 1. Let $(C, A)$ be observable. Then, there exists $K \in \mathbf{R}^{n}$ such that all $F_{i}, i=1, \ldots, m$, are stable.
Proof. Set $\operatorname{det}(\lambda I-A)=\lambda^{n}+\alpha_{1} \lambda^{n-1}+\cdots+\alpha_{n}$ as the characteristic polynomial of $A$. Define the vectors $u_{1}=\left(1 \alpha_{1} \ldots \alpha_{n-1}\right)^{\mathrm{T}}, u_{2}=\left(01 \alpha_{1} \ldots \alpha_{n-2}\right)^{\mathrm{T}}, \ldots, u_{n}$ $=(00 \ldots 1)^{\mathrm{T}}$, and define the matrices $U=\left(u_{1} u_{2} \ldots\right.$ $\left.u_{n}\right), R=U Q$, where $Q$ is given by (7). By using simple algebra and Cayley-Hamilton theorem (i.e., $\left.A^{n}+\alpha_{1} A^{n-1}+\cdots+\alpha_{n} I=0\right)$ and (6) we obtain:

$R A R^{-1}=\left(\begin{array}{ccccc}-\alpha_{1} & 1 & 0 & \ldots & 0 \\ -\alpha_{2} & 0 & 1 & \ldots & 0 \\ & & \vdots & & \\ -\alpha_{n-1} & 0 & 0 & \ldots & 1 \\ -\alpha_{n} & 0 & 0 & \ldots & 0\end{array}\right), \quad C R=C$.

Let us define $\hat{h}_{i}=R h_{i}=\left(\hat{h}_{i 1} \ldots \hat{h}_{i n}\right)^{\mathrm{T}}, \hat{K}=R K=$ $\left(\hat{k}_{1} \ldots \hat{k}_{n}\right)^{\mathrm{T}}$, and set $\hat{F}_{i}=R F_{i} R^{-1}, i=1, \ldots, m$. By using (17) we obtain the following characteristic polynomial for $\hat{F}_{i}$

$$
\begin{aligned}
\operatorname{det}\left(\lambda I-\hat{F}_{i}\right)= & \lambda^{n}+\left(\hat{k}_{1}-\gamma_{i 1}\right) \lambda^{n-1} \\
& +\left(\hat{k}_{2}-\gamma_{i 2}\right) \lambda^{n-2}+\cdots+\left(\hat{k}_{n}-\gamma_{i n}\right),
\end{aligned}
$$

where $\gamma_{i j}=\hat{h}_{i j}-\alpha_{j}, i=1, \ldots, m, j=1, \ldots, n$. By using Routh criterion, after some straightforward algebra it could be shown that given $\gamma_{i j}$, one can find the weights $\hat{k}_{j}$ such that (18) yields stable polynomials for $i=1, \ldots, m$. For example, for $n=2$, it suffices to choose

$\hat{k}_{1}>\max _{i}\left\{\gamma_{i 1}\right\}, \quad \hat{k}_{2}>\max _{i}\left\{\gamma_{i 2}\right\}$,

and for $n=3$, it suffices to choose

$\hat{k}_{1}>\max _{i}\left\{\gamma_{i 1}\right\}, \quad \hat{k}_{3}>\max _{i}\left\{\gamma_{i 3}\right\}$,

$\hat{k}_{2}>\max _{i}\left\{\gamma_{i 2}+\left(\hat{k}_{3}-\gamma_{i 3}\right) /\left(\hat{k}_{1}-\gamma_{i 1}\right)\right\}$.

Since $F_{i}$ and $\hat{F}_{i}$ are similar, they have the same eigenvalues, hence all $F_{i}$ are stable as well. The required gain is $K=R^{-1} \hat{K}$.

Note that although each $F_{i}$ in (16) is stable with a proper choice of $K$, this does not necessarily imply that the resulting error is also stable. This is due to switching in (16), and there are some examples which indicate that switching between stable systems may cause instability in certain cases, see [17]. In such examples, the instability is often due to a particular (often periodic) switching. When applied to chaotic systems, as in this work, the switching is also chaotic. 
In such cases it is reasonable to expect that chaotic switching between stable systems as in (16) might yield stable error dynamics. However, this conjecture requires further investigation. The necessary and sufficient conditions on $F_{i}$ to guarantee the stability of (16) independent of the switching pattern are not known. Some preliminary results indicate that the problem might even be undecidable, see [17]. Some sufficient conditions to guarantee the stability exist in the literature. The simplest one is the existence of a common quadratic Lyapunov function $V=e^{\mathrm{T}} P e$ for (16), where $P$ is a symmetric and positive definite matrix, i.e., there exist symmetric and positive definite matrices $P, Q_{i}$ such that $F_{i}^{\mathrm{T}} P+P F_{i}=-Q_{i}$ holds for $i=1, \ldots, m$. Indeed, in this case, by differentiating $V=e^{\mathrm{T}} P e$, and using (16) we obtain $\dot{V}=-e^{\mathrm{T}} Q_{i} e<$ 0 , and by using standard Lyapunov stability arguments, we conclude the asymptotic stability of error. In fact, it can easily be concluded that the decay is exponential in this case. Unfortunately, the necessary and sufficient conditions for the existence of such a common Lyapunov function is also not known. A sufficient condition is the existence of a common set of eigenvectors $\left\{v_{1} \ldots v_{n}\right\}$ for $F_{i}$, i.e., $F_{i} v_{j}=\lambda_{i j} v_{j}$ holds for $i=1, \ldots, m, j=1, \ldots, n$. Indeed, in this case, if we set $V=\left(v_{1} \ldots v_{n}\right)$, after some straightforward calculations it is easy to show that $P=\left(V V^{\mathrm{T}}\right)^{-1}$ yields a common Lyapunov function. The required condition may seem to be restrictive, but it holds in certain cases, including our first simulation example given in the next section.

Finally we note on the robustness of the synchronization scheme proposed in this Letter. If the error dynamics given by (14) or (16) is exponentially stable, then the corresponding scheme is robust with respect to noise and parameter mismatch. This claim can be justified by using the robustness of exponentially stable systems, see, e.g., [2,6]. As an example, consider the synchronization scheme given by (15) and assume that the synchronization signal is corrupted by noise. In this case, $z_{1}$ in (15) should be replaced by $z_{1}+n$, where $n$ represents the noise. The corresponding error dynamics now will be as follows:

$\dot{e}=F_{i} e-K n$.

Hence, the noise $n$ enters as an input into an exponentially stable system, and if $n$ is bounded it produces a bounded error as well. To elaborate further, let us as- sume that $V=e^{\mathrm{T}} P e$ is a common Lyapunov function for (16). By differentiating $V$, using $F_{i}^{\mathrm{T}} P+P F_{i}=$ $-Q_{i}$ and (21), we obtain:

$\dot{V}=-e^{\mathrm{T}} Q_{i} e+2 e^{\mathrm{T}} P K n$.

Now assume that the noise is bounded and set $n_{\max }=$ $\max _{t}|n(t)|$. Let $\lambda_{\min }(\cdot)$ and $\lambda_{\max }(\cdot)$ denote the minimum and maximum eigenvalues of symmetric matrices, respectively, and set $\alpha=\lambda_{\max }(P), \quad \beta=$ $\min _{i}\left\{\lambda_{\min }\left(Q_{i}\right)\right\}$. From (22) we obtain

$\dot{V} \leqslant-\beta\|e\|^{2}+2 \alpha n_{\max }\|K\|\|e\|$,

where $\|\cdot\|$ denotes the standard Euclidean norm. By using standard invariance arguments, see [18], it is easy to deduce that the error will be bounded by $e_{\infty}=$ $2 \alpha\|K\| n_{\max } / \beta$ as $t \rightarrow \infty$. Note that the noise affects the error linearly. The same also holds for the gains. Hence, larger gains are undesirable since they amplify the effect of noise as well, as is expected.

\section{Simulation results}

In this section, we present two simulation examples. The first one is called the generalized Chua's circuit, and is given by the following equations:

$\dot{z}_{1}=\alpha\left[z_{2}-h\left(z_{1}\right)\right]$,

$\dot{z}_{2}=z_{1}-z_{2}+z_{3}$,

$\dot{z}_{3}=-\beta z_{2}$,

where the nonlinearity $h(\cdot)$ is given by the following piecewise-linear characteristic

$$
\begin{aligned}
h\left(z_{1}\right)=m_{2 q-1} z_{1}+\sum_{j=1}^{2 q-1}( & \left(m_{j-1}-m_{j}\right) \\
& \times\left(\left|z_{1}+c_{j}\right|-\left|z_{1}-c_{j}\right|\right) .
\end{aligned}
$$

Here $\alpha, \beta, q, m_{k}$ and $c_{l}$ denote various coefficients. For various values of these coefficients, this system exhibits various chaotic behaviours, see, e.g., [19]. In our simulations, we chose $\alpha=9, \beta=14.286, q=2$, $m_{0}=0.9 / 7, m_{1}=-3 / 7, m_{2}=3.5 / 7, m_{3}=-2.4 / 7$, $c_{1}=1, c_{2}=2.15, c_{3}=4$, and for these parameters this system is known to exhibit a three-scroll chaotic behaviour, as shown in Fig. 1(a); for details and also for an electronic implementation of the relevant 


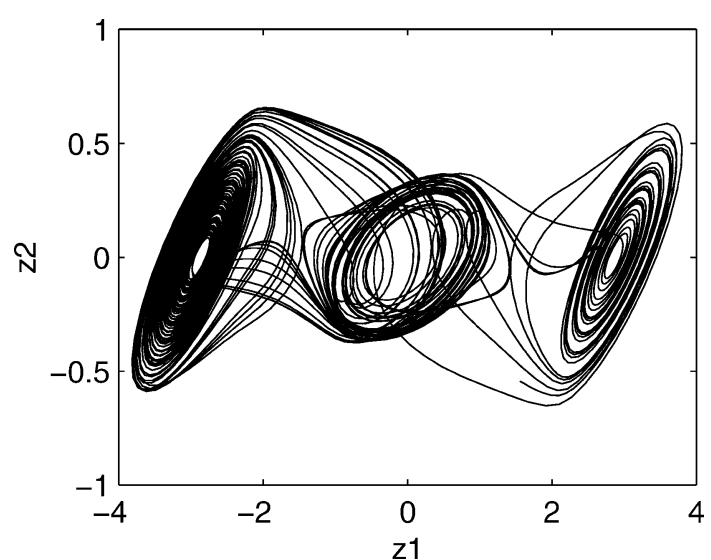

(a)

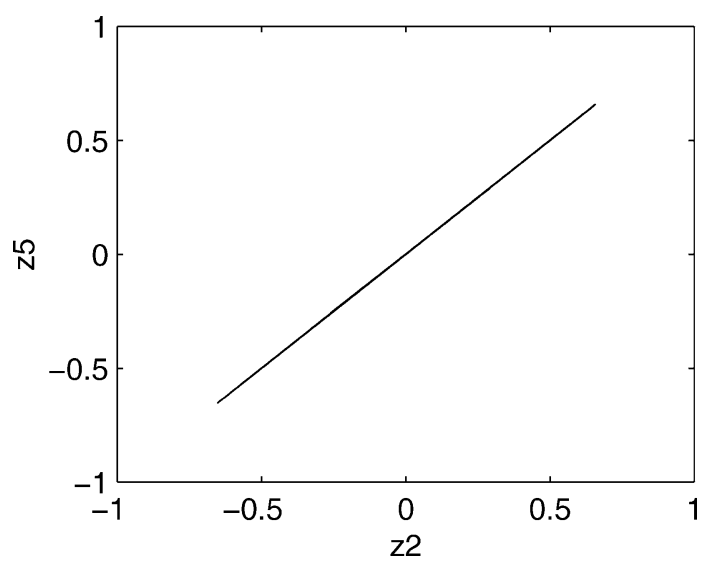

(c)

Fig. 1. Simulation results for the generalized Chua's circuit. (a) $z_{1}$ vs. $z_{2}$. (b) $z_{1}$ vs. $z_{4}=\hat{z}_{1}$. (c) $z_{2}$ vs. $z_{5}=\hat{z}_{2}$. (d) $\|e\|$ vs. time.

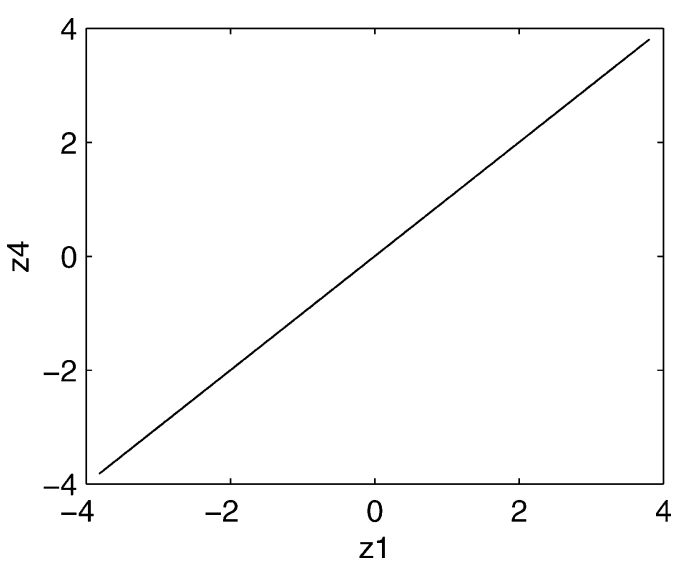

(b)

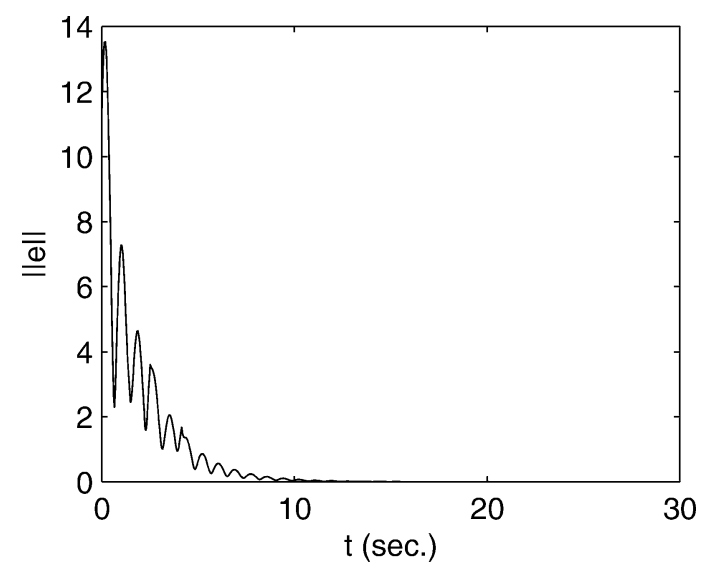

(d)

where

$d_{1}=\left[-m_{0} c_{1}+m_{1}\left(c_{1}-c_{2}\right)+m_{2}\left(c_{2}-c_{3}\right)+m_{3} c_{3}\right]$,

$d_{2}=\left[-m_{0} c_{1}+m_{1}\left(c_{1}-c_{2}\right)+m_{2} c_{2}\right]$,

$d_{3}=\left[c_{1}\left(m_{1}-m_{0}\right)\right]$.

In the formalism of Theorem 1 , we have $\alpha_{1}=1$, $\alpha_{2}=\beta-\alpha, \alpha_{3}=0$, and the required transformation in (17) is given as $R=\left(r_{1} r_{2} r_{3}\right)$ with $r_{1}=(11 \beta)^{\mathrm{T}}$, $r_{2}=(0 \alpha 0)^{\mathrm{T}}, r_{3}=(00 \alpha)^{\mathrm{T}}$. By using the coefficients given above and (20), we obtain $\hat{k}_{1}>2.86, \hat{k}_{3}>$ 55.103 , and remaining gain $\hat{k}_{2}$ can be found from (20). If we choose $\hat{k}_{1}=4, \hat{k}_{3}=57.144$, we obtain $\hat{k}_{2}>$ 5.842; and with the selection of $\hat{k}_{2}=13$, we obtain the gain vector $K=R^{-1} \hat{K}=(410)^{\mathrm{T}}$. In this case all matrices $F_{i}=A_{i}-K C$ are stable; further- 


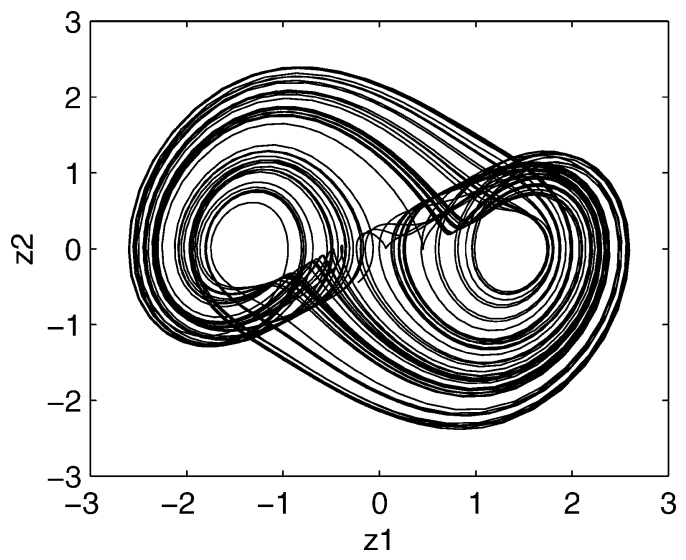

(a)

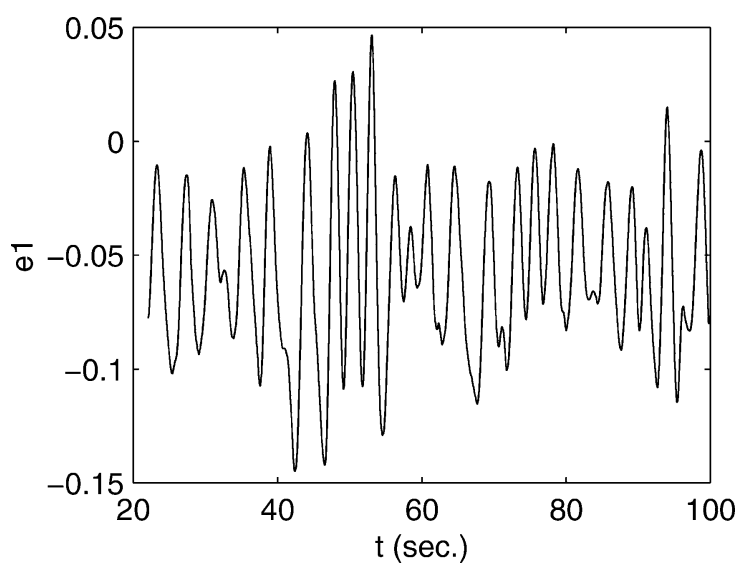

(c)

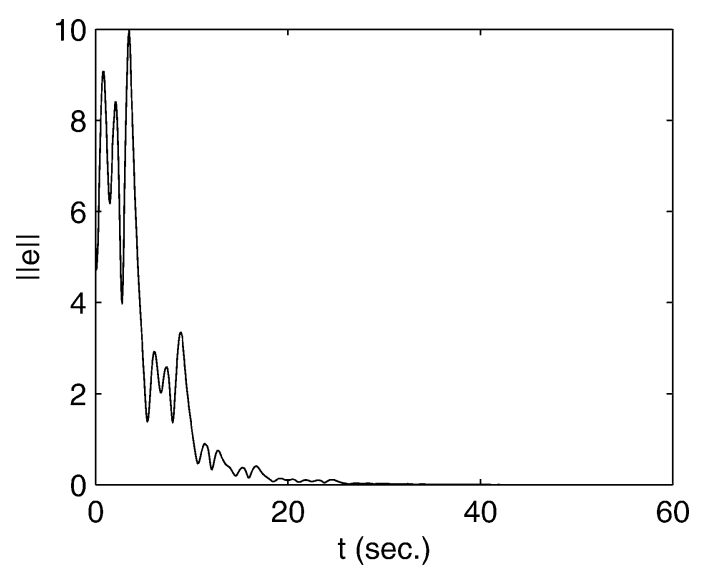

(b)

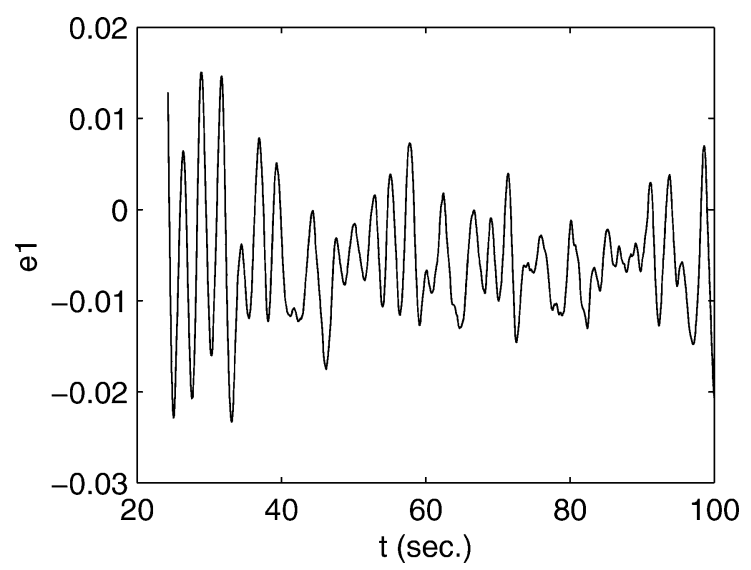

(d)

Fig. 2. Simulation results for the Brockett system. (a) $z_{1}$ vs. $z_{2}$. (b) $\|e\|$ vs. time. (c) $e_{1}=z_{1}-\hat{z}_{1}$ vs. time for $a=0.2$. (d) $e_{1}=z_{1}-\hat{z}_{1}$ vs. time for $a=0.02$.

more, direct calculation shows that all $F_{i}$ have a common set of eigenvectors, hence a common Lyapunov function exists. Therefore, we conclude that the error dynamics given by (16) is exponentially stable. We simulated the observer structure given by (15) together with the given system (24)-(27), and the simulation results are shown in Fig. 1. The initial conditions are chosen as $z(0)=(41-4)^{\mathrm{T}}, \hat{z}(0)=-z_{0}$, hence $\|e(0)\|=\|z(0)-\hat{z}(0)\|=11.48$, which is quite large. In Figs. 1(b) and (c), the plots of $z_{1}$ versus $\hat{z}_{1}$ and $z_{2}$ versus $\hat{z}_{2}$ are shown, respectively (note that in the figures we used $z_{4}=\hat{z}_{1}, z_{5}=\hat{z}_{2}$ ). Also note that these figures are plotted after the transients. Finally, in Fig. 1(d), we plotted the synchronization error magnitude $\|e\|$.
In the second simulation, we used the Brockett system, see [6] for details. This system is in Lur'e form and could be transformed into the structure given by (1)-(5) as described in Section 2. Here we have $m=3, k_{1}=-k_{2}=-1$. By using

$$
A=\left(\begin{array}{ccc}
0 & 1 & 0 \\
0 & 0 & 1 \\
0 & -1.25 & -1
\end{array}\right), \quad b=\left(\begin{array}{l}
0 \\
0 \\
1
\end{array}\right),
$$

we can transform the Brockett system into the structure given by (1)-(5) with $h_{1}=h_{3}=-3.6 b, h_{2}=$ $1.8 b, b_{1}=-b_{3}=-5.4 b, b_{2}=0$. This system exhibits chaotic behaviour as shown in Fig. 2(a). To calculate the required gain vector in (15), we use the procedure given in Theorem 1. In the formalism of Theorem 1, 
we have $\alpha_{1}=1, \alpha_{2}=1.25, \alpha_{3}=0$, and the required transformation in (17) is given as $R=\left(r_{1} r_{2} r_{3}\right)$ with $r_{1}=\left(\begin{array}{llll}1 & 1 & 1.25\end{array}\right)^{\mathrm{T}}, r_{2}=\left(\begin{array}{lll}0 & 1 & 1\end{array}\right)^{\mathrm{T}}, r_{3}=\left(\begin{array}{lll}0 & 0 & 1\end{array}\right)^{\mathrm{T}}$. By using the coefficients given above and (20), we obtain $\hat{k}_{1}>-1, \hat{k}_{3}>1.8$, and remaining gain $\hat{k}_{2}$ can be found from (20). If we choose $\hat{k}_{1}=0, \hat{k}_{3}=2$, we obtain $\hat{k}_{2}>4.35$; and with the selection of $\hat{k}_{2}=4.5$, we obtain the gain vector $K=R^{-1} \hat{K}=(04.5-2.5)^{\mathrm{T}}$. In this case all matrices $F_{i}=A_{i}-K C$ are stable. However, unlike the previous case the matrices $F_{i}$ do not have a common set of eigenvectors, hence existence of a common Lyapunov function is not guaranteed in this case. Since the necessary and sufficient conditions for the existence of a common Lyapunov function are not known yet, we do not claim the nonexistence of a common Lyapunov function as well. We simulated the system given by (2)-(5) and the observer given by (15). The resulting error in synchronization is given in Fig. 2(b). As can be seen from the figure, the error system is exponentially stable. Hence, as mentioned in Section 2, this system should be robust with respect to noise. To demonstrate this point, we also assumed that the synchronization signal $z_{1}(t)$ is corrupted with a noise term $n(t)$, i.e., in (15) we used $z_{1}+n$. The noise is assumed to be random and uniformly distributed in [0a], where $a>0$ is a constant. We considered the case $a=0.2$ and $a=0.02$, and the resulting synchronization errors are shown in Figs. 2(c) and (d), respectively. Both figures are plotted after the transients. Note that since the peak amplitude of $z_{1}$ is around 2, the case $a=0.2$ corresponds to a $10 \%$ perturbation, and as can be seen in Fig. 2(c), the synchronization error magnitude is of the same order of $a$. The case $a=0.02$ corresponds to $1 \%$ perturbation, and as can be seen in Fig. 2(d), the synchronization error magnitude is still of the same order of $a$. For the Brockett system, the initial conditions are chosen as $z(0)=(21-1)^{\mathrm{T}}, \hat{z}(0)=-z_{0}$, hence $\|e(0)\|=\|z(0)-\hat{z}(0)\|=4.89$, which is quite large. These simulations show the effectiveness of the proposed technique.

\section{Conclusion}

In this Letter we considered a special class of chaotic systems, which are given by piecewise-linear dynamics. This class of systems, although seems to be somehow restricted, contains some meaningful classes of chaotic systems, including most of the chaotic electronic oscillators, already proposed in the literature. We proposed two observer-based synchronization schemes for this class of systems. We note that by using general observer-based designs different observer-based schemes may also be constructed for such systems, see, e.g., [2,6]. However, in such designs the properties of the drive system are not fully utilized, and as a result the required gain vector to guarantee the synchronization may be quite high, see, e.g., [8]. In our approach, the drive system characteristics for the class of systems under consideration are incorporated into the observer design, and hence the required gains to guarantee the synchronization are expected to be small. We prove various synchronization results and comment on the robustness of the proposed schemes. We also presented some simulation results which show the effectiveness of the proposed schemes.

We also note that the proposed observer has a switching behaviour, resulting from the piecewiselinear dynamics of the drive system. Due to this structure, the control action in the observer can be interpreted as some "if-then" rules. This interpretation may be used in designing, or in incorporation of fuzzy control techniques into the synchronization schemes. However, this point requires further investigation.

\section{References}

[1] L.M. Pecora, T.L. Carroll, Phys. Rev. Lett. 64 (1990) 821.

[2] Ö. Morgül, E. Solak, Phys. Rev. E 55 (5) (1996) 4803.

[3] G. Chen, Control and synchronization of chaotic systems (a bibliography), ECE Dept., Univ. of Houston, TX, available from ftp://ftp.egr.uh.edu/pub/TeX/chaos.tex (login name "anonymous", password: your email address).

[4] G. Chen, X. Dong, From Chaos to Order: Methodologies, Perspectives and Applications, World Scientific, Singapore, 1998.

[5] Ö. Morgül, M. Feki, Phys. Lett. A 251 (1999) 169.

[6] Ö. Morgül, E. Solak, Int. J. Bifurcation Chaos 7 (6) (1997) 1307.

[7] Ö. Morgül, Phys. Rev. Lett. 82 (1) (1999) 77.

[8] Ö. Morgül, E. Solak, Phys. Lett. A 279 (2001) 47.

[9] Ö. Morgül, Phys. Lett. A 262 (1999) 144.

[10] C.C. Fuh, P.C. Tung, Phys. Lett. A 229 (1997) 228.

[11] Ö. Morgül, Electronics Lett. 31 (24) (1995) 2058.

[12] A.S. Elwakil, M.P. Kennedy, IEEE Trans. Circuits Syst. Part 147 (4) (2000) 582. 
[13] L.O. Chua, C.W. Wu, A. Huang, G.Q. Zhong, IEEE Trans. Circuits Syst. Part 140 (1993) 722.

[14] Ö. Morgül, IEEE Trans. Circuits Syst. Part 147 (9) (2000) 1424.

[15] Z. Li, J.B. Park, G. Chen, Y.H. Joo, Int. J. Bifurcation Chaos (2001), accepted.

[16] J.Q. Fang, Y. Hong, G. Chen, Phys. Rev. E 59 (3) (1999) 2523.
[17] E. Solak, Observability and Observers for Nonlinear and Switching Systems, PhD Thesis, Bilkent University, Ankara, Turkey, 2001.

[18] H.K. Khalil, Nonlinear Systems, 2nd edn., Macmillan, New York, 1996.

[19] M.E. Yalçın, J.A.K. Suykens, J. Vandewalle, IEEE Trans. Circuits Syst. Part 147 (3) (2000) 425. 